\title{
Atomic force microscopy combined with confocal laser scanning microscopy: a new look at cells
}

\section{Constant A J Putman, Anne Marie van Leeuwen, Bart G de Grooth, Katarina Radošević, Kees $O$ van der Werf, Niek F Van Hulst and Jan Greve}

Department of Applied Physics, University of Twente, PO Box 217, 7500 AE Enschede, The Netherlands

Received 16 October 1992, in final form 2 November 1992

\begin{abstract}
A stand-alone atomic force microscope (AFM) has been developed, which features a large scan area and which allows operation under liquid. This system was combined with a confocal laser scanning microscope (CLSM). Information about cell structures, obtained by CLSM, can be complemented with images of the cell surface obtained with the AFM. This is illustrated by studying the pseudopodia of cells from a human cell line (K562-cells, predecessor of erythroblasts) and the cytoskeleton of monkey kidney cells (in air and under liquid), both stained with F-actin-specific fluorescent probes. Images of the cytoskeleton during the cytotoxic interaction between a natural killer and a K562 target cell are presented. Our results show that combination of these techniques can provide new information about cells and cellular structures.
\end{abstract}

Keywords: atomic force microscopy, confocal laser scanning microscopy, cytoskeleton, cytotoxic interaction.

\section{Introduction}

In atomic force microscopy (AFM) [1] a very sharp probe is raster-scanned in close proximity to the sample surface under investigation. The sharp probe is mounted on a weak cantilever. In contact AFM (also called static AFM) repulsive forces between atoms at the tip of the probe and atoms at the sample surface will deflect the cantilever. Measuring the deflection of the cantilever while monitoring the sample position during rasterscanning with a piezo-electric $x y z$-translator, a topographic image of the surface can be obtained.

AFM can give high-resolution images, atomic and molecular resolution images have been obtained on a wide variety of samples in air and under liquid, and has been applied to a growing number of biological samples (for a review see Hoh and Hansma [2]). Despite the fact that AFM is essentially a surface technique, it is possible to obtain sub-surface information. Keller et al [3] have show that the AFM is capable of obtaining structural information on the cytoskeleton lying just beneath the membrane with a resolution higher than with the optical microscope. The membrane is smoothly draped over the fibers constituting the cytoskeleton and a corrugation is created which enables indirect imaging of the cytoskeleton lying directly underneath the membrane. It can be called indirect imaging, because the membrane is between the tip and the cytoskeleton. In a recent study, Henderson et al [4] showed that it is possible to monitor the dynamics of actin filaments in living glial cells. At the cell margin of these large cells $(>100 \mu \mathrm{m})$ the lamellipodium spreads and attaches in a flattened form to the substrate. Because of this flattened morphology, merely consisting of actin filaments covered by the cell membrane, it is possible to study the cytoskeleton.

In confocal laser scanning microscopy (CLSM) [5] biological specimens stained with a fluorescent probe can be studied in a three-dimensional way by reconstructing a series of focal sections through the object. The resolution is limited by diffraction to about $200 \mathrm{~nm}$ in the $x y$-plane and $650 \mathrm{~nm}$ in the $z$-direction (wavelength: $600 \mathrm{~nm}$, numerical aperture: 1.4).

It would be advantageous to combine these two techniques in a single instrument, which enables one to obtain images with the AFM and the CLSM simultaneously. With a conventional AFM this is not possible, because the sample is not accessible for optical imaging with a high numerical aperture objective. The solution 
would be to use a so-called stand-alone AFM, which scans the tip instead of the sample. At the basis of this concept lies the idea that with a scanning tip samples of any kind and size can be imaged. More interesting for biological applications is the fact that such a stand-alone AFM can be combined with an optical microscope featuring epi-illumination. The concept of a stand-alone AFM has been described by Hipp et al [6], Putman et al [7] and Sarid et al [8]. Unfortunately all of these systems have their specific drawbacks. In short: Hipp et al and Putman et al use a fixed laser beam to sense the cantilever displacement through angular deflection of the laser beam [9] resulting in a limited scan range $\left(5 \times 5 \mu \mathrm{m}^{2}\right)$. The system of Sarid et al, in which laser diode and cantilever are both scanned by the piezo tube, utilizes optical feedback into a laser diode for tip displacement detection. As a consequence this AFM cannot be operated in liquid.

For those reasons we have designed a stand-alone AFM, which combines features of the two types of stand-alone AFMs mentioned above. Optical beam deflection [9], less troublesome than optical feedback, is used for displacement detection of the cantilever. This makes it possible to operate the AFM in a liquid environment. The laser diode and cantilever are scanned simultaneously by the piezo tube. Subsequently the scan range of this AFM is not limited by the dimensions of the cantilever and/or the waist of the laser beam, but solely determined by the characteristics of the piezo tube.

In this paper we present a system combining the home-built stand-alone AFM and a commercially available CLSM. It is now possible to study the internal structures of a cell with the CLSM and complement this with high-resolution topographic information obtained with the AFM in one instrument.

\section{Materials and methods}

\subsection{Sample preparation}

K562-cells, predecessors of erythroblasts, were maintained as previously described [10]: NK-cells, clone T6, were grown as described elsewhere [11]. Equal numbers of NK- and K562-cells were mixed, centrifuged for $5 \mathrm{~min}$ at $200 \mathrm{~g}$ and incubated for $30 \mathrm{~min}$ at $37^{\circ} \mathrm{C}$. After incubation cells were plated on $0.01 \%$ poly-L-lysinetreated cover glasses and allowed to attach for about $15 \mathrm{~min}$ at room temperature. The cells were fixed with $3.7 \%$ PFA/PBS (Merck). permeabilized with $0.1 \%$ Triton $\mathrm{x}-100 / \mathrm{PBS}$ (Sigma) and stained with BODIPY 581/591 Phalloidin (Molecular Probes Inc., OR) to visualize F-actin. After rinsing with PBS and distilled water, samples were air dried and analysed.

Monkey kidney cells were grown on a coverglass at $37^{\circ} \mathrm{C}$ in IMDM (Iscove's Modified Dulbecco's Medium) supplemented with $25 \mathrm{mM}$ HEPES, $10 \%$ Foetal Calfserum, $100 \mu \mathrm{g}$ streptomicin $/ \mathrm{ml}, 100$ units penicillin $/ \mathrm{ml}$ and $2 \mathrm{mM}$ L-glutamine. Fixation and staining were the same as described above for the K562- and NK-cells. Some of the samples were rinsed with PBS and air dried. Others were stored in PBS.

\subsection{Atomic force microscope combined with confocal laser scanning microscope}

Figure 1(a) shows a schematic representation of the stand-alone AFM. At the end of the piezo tube both cantilever and laser diode are attached. The laser diode and focusing lens are positioned on an adjustable plate inside the piezo tube (inner diameter of $17 \mathrm{~mm}$ ), which allows one to manipulate the laser beam at the cantilever (see blow-up in figure $1(b)$ ). Two flexible PVC rods form the connection between adjustment knobs (\#1 in figure $1(a)$ ) and screws in the adjustable plate (\#13 in figure $1(b)$ ). The cantilever is fixed to the tip holder by a steel wire. The problem of limited scan range is solved because tip and laser diode are scanned simultaneously: the laser spot stays on the cantilever while scanning. The scan range is solely determined by the characteristics of the piezo tube. Operation in liquid of the design as described will be erroneous due to disturbances at the air-liquid interface. Attaching a small piece of plexiglass to the tip holder just above the cantilever (plexiglass not shown in figure $1(b)$ ) creates a stable glass-water interface, acting as an optical window for the laser beam and thus allowing operation in liquid.

The beam reflected off the back of the microfabricated cantilever with integrated pyramidal tip $[12,13]$ is directed to a segmented photodiode by a tunable mirror. A displacement of the cantilever results in a shift of the laser beam at the photodiode. This gives a difference signal between the two parts of the photodiode, which is proportional to the height variations at the sample surface. This detection scheme, called optical beam deflection [9], can be as sensitive for displacement detection as interferometry [14]. Three micropositioning screws form a tripod construction for a stable contact with the baseplate. A reduction factor of 15 (through the mechanical lever formed by the three screws and the position of the tip) allows for a gentle manual approach of the tip towards the sample surface. Feedback electronics keep the applied force between sample and tip at an average value of about $10^{-8} \mathrm{~N}$ in air and $10^{-9} \mathrm{~N}$ in liquid [15]. The $\mathrm{Si}_{3} \mathrm{~N}_{4}$-cantilevers used are $\mathrm{V}$-shaped, $200 \mu \mathrm{m}$ long and have a force constant of $0.032 \mathrm{~N} \mathrm{~m}^{-1}$ (specifications from the manufacturer [13]). The imaging time ranges from 75 to $150 \mathrm{~s}$ per image and the maximum scan range is $24 \times 24 \mu \mathrm{m}^{2}$. The AFM is calibrated using a cross-grating with a known spacing ( $x$ - and $y$ directions) and the optical beam deflection method (z-direction). 

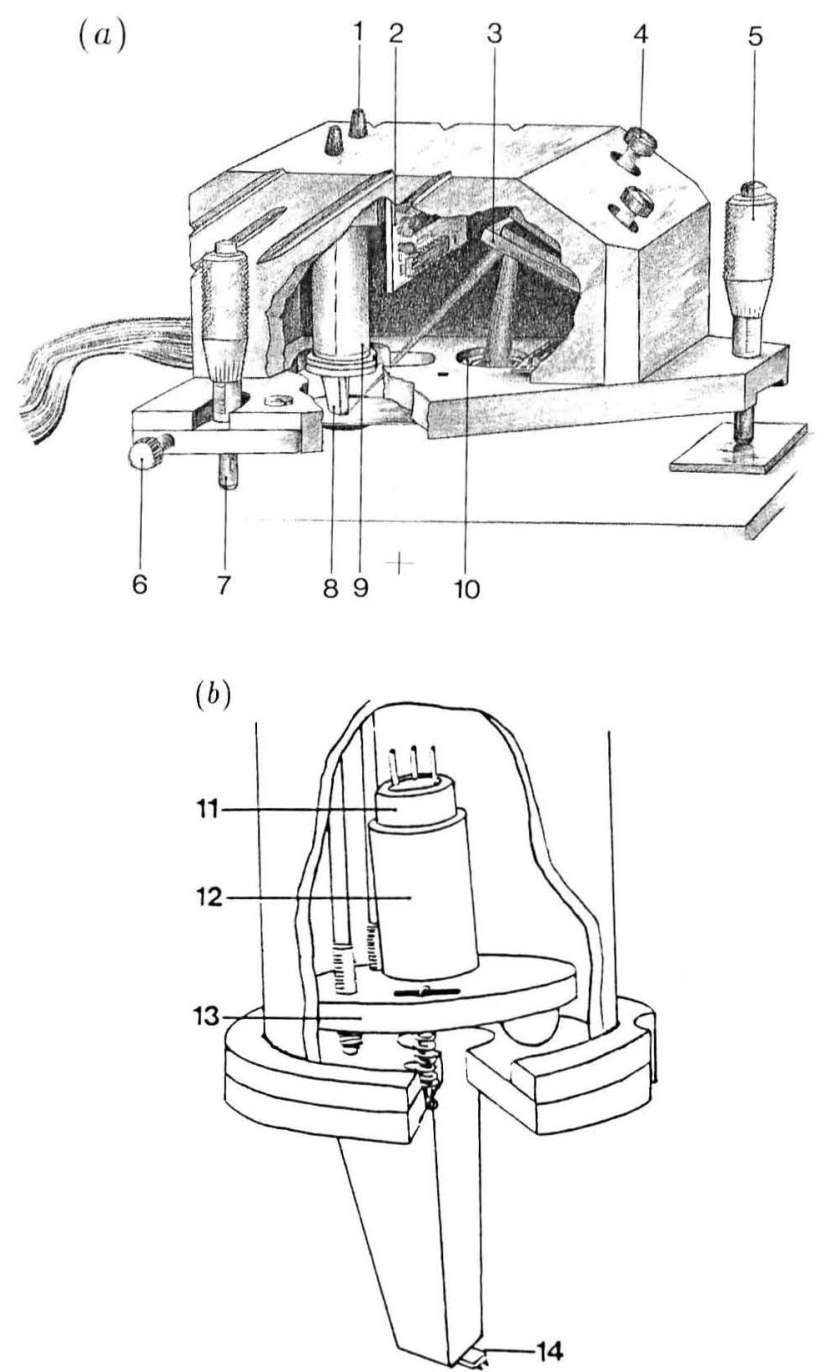

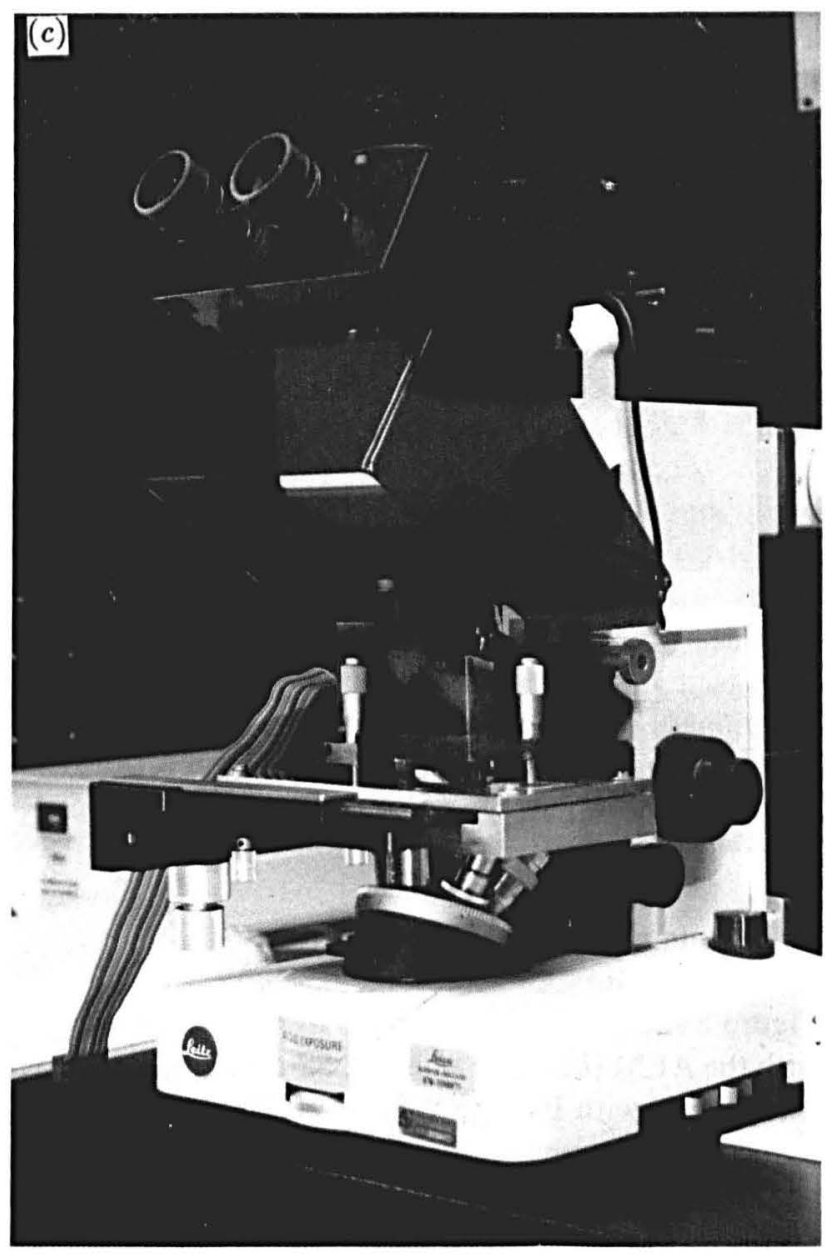

Figure 1. The stand-alone AFM. (a) Overall layout, $(b)$ blow-up of essential part. The numbers indicate the following parts: (1) knobs for adjustment of the laser beams at the cantilever; (2) pre-amplifier electronics; (3) mirror; (4) adjustment knobs of the mirror; (5) micrometer screw for fine approach; (6) mount for $x y$-movement of the AFM to get the tip in line with the inverted optical microscope; (7) micrometer screw for coarse approach; (8) tip holder with a steel wire to hold the cantilever with tip in place; (9) piezo-electric tube for xyz-scanning; (10) photodiode; (11) laser diode; (12) focusing lens; (13) adjustable plate; (14) cantilever. (c) A photograph of the stand-alone AFM combined with CLSM. The scanning sample stage is positioned between the base plates of the optical microscope and the AFM.

When displaying the applied voltages to the $z$ electrode of the piezo tube, a calibrated height image of the object under investigation is created (also called the height mode). The feedback loop, however, is not capable of compensating for fast changes in topography at the sample surface. In the error signal mode [16] used in this paper, the difference signal from the photodiode not compensated by the feedback loop is displayed. In short: the height mode shows the low-pass filtered and the error signal mode the high-pass filtered image of the surface topography, in which the cutoff frequency is determined by the piezo tube characteristics and the feedback electronics. In the error signal mode the up-slope of a surface feature becomes light and the down-slope dark. Unless mentioned otherwise, the AFM images presented show raw data.
The stand-alone AFM (dimensions: $80 \times 80 \times 110$ $\mathrm{mm}^{3}$ ) is combined with a CLSM from Leica Lasertechnik (Heidelberg, Germany) [17]. The CLSM is equipped with an inverted fluorescence microscope (Leica Fluovert FU) and an air-cooled argon-krypton laser of which different lines can be selected for excitation. The CLSM uses a scanning mirror which shifts the laser beam in the $x y$-plane and a computer controlled stage, moveable in the $z$-direction with $40 \mathrm{~nm}$ resolution. A variable pinhole in front of the photomultipliers is used to balance between confocal resolution and the signal-to-noise ratio of the fluorescent signal $[18,19,20]$. For the excitation of BODIPY 581/591 Phalloidin the $568 \mathrm{~nm}$ line was selected and a long-pass filter $(580 \mathrm{~nm})$ was inserted in the fluorescence detection path. Series of confocal sections, $0.33 \mu \mathrm{m}$ apart, were scanned, each image 
averaged by 8 to 16 line scans. After calculation of the 3D data by a software function called 'Simulated Fluorescence Process' [21], the resulting image is displayed in a 2D field. For the sample imaged under liquid one confocal section was taken, line scanned and averaged 64 times.

An extra base plate, which serves as a support for the AFM, has to be mounted on the CLSM. The scanning sample stage is sandwiched between the base plate of the CLSM and the one on which the AFM is placed (see figure 1(c). Sample and tip can be viewed simultaneously by the optical microscope or by the CLSM. The object of interest can be positioned under the AFM tip using the $x y$-sample stage. Initial measurements with the AFM indicated vibrations of the sample stage with an amplitude of about $100 \mathrm{~nm}$ in the 70 to $100 \mathrm{~Hz}$ range. By immobilizing the sample stage with screws, after imaging the object with the CLSM, the vibrations could be reduced to an amplitude between 5 and $10 \mathrm{~nm}$.

\section{Results and discussion}

Figure 2 shows images obtained with the CLSM $(a)$ and with the AFM $(b)$ of the same K562-cell, in which F-actin was stained with BODIPY 581/591 Phalloidin. A strong correlation between features in both images is apparent: all the pseudopodia observed with the AFM are also visible in the fluorescence image, indicating the presence of F-actin in all the pseudopodia. The height of the

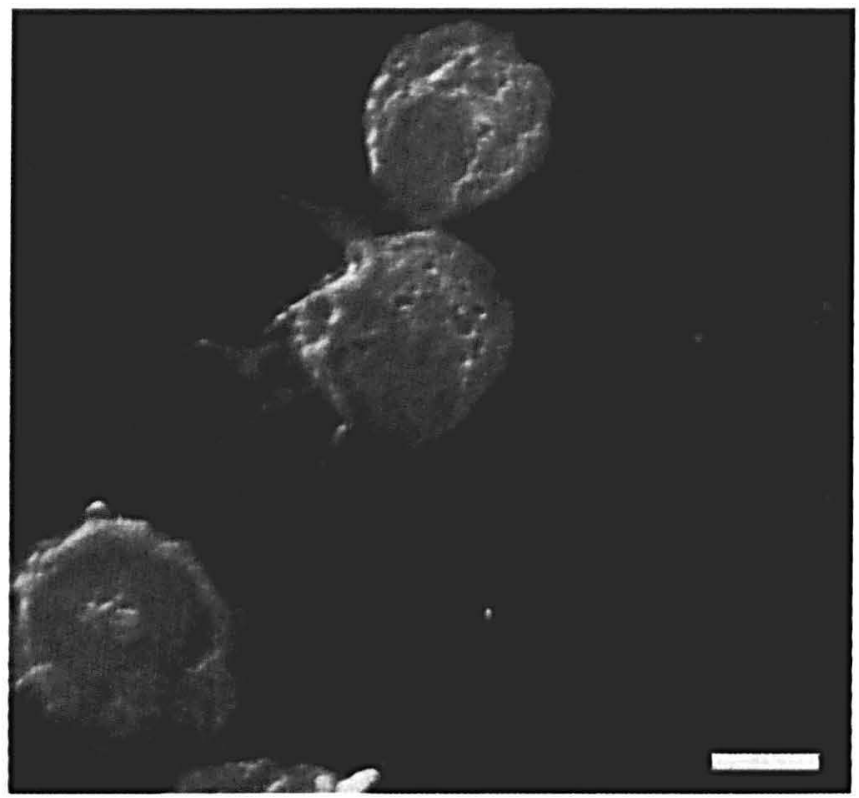

(a) pseudopodia, obtained from the AFM image, is in the $50-100 \mathrm{~nm}$ range. This is in correspondence with earlier observations on this type of cell with the AFM [22]. On the cell membrane (figure $2(b)$ ) some 'craters' are present. The origin of these structures is yet unknown.

Figure 3(a) shows a confocal image of a conjugate of a K562- and NK-cell during the cytotoxic process: the membrane at the top of the K562-cell is totally disrupted. This is confirmed by images obtained with the AFM (not shown here), which show in that region a vague morphology not resembling the normal appearance of a cell membrane. At the position indicated by the arrow, an AFM image has been obtained. The AFM image (figure $3(b)$ ) shows a thin microspike originating from the $\mathrm{K} 562$-cell, which is not visible in the confocal image. It runs away from the cell over a distance of more than $40 \mu \mathrm{m}$ before the microspike terminates (not shown here). Since the fluorescent probe used is specific for F-actin, this difference indicates that $\mathrm{F}$-actin is not present. In most cases the correspondence between CLSM and AFM image is high, but as the images in figure 3 show large differences are also observed. This illustrates that with the combination of AFM and CLSM more complete information is obtained.

In figure 4 the contact between NK- and K562-cell in a conjugate is visualized. Again, craters are visible on the K562-cell. A magnified image of the contact area is shown in figure $4(c)$. Finger-like structures extending from the NK-cell onto the glass surface (figure 4(b)), seem to be present also in the contact area with the K562-cell.

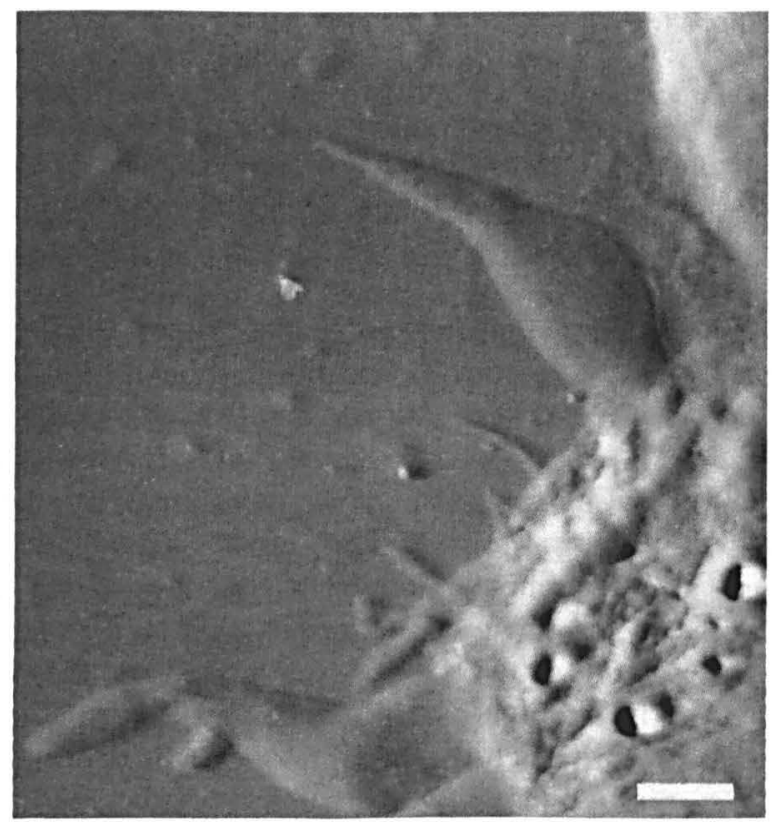

(b)

Figure 2. Images of a K562-cell stained with BODIPY 581/591 Phalloidin recorded with $(a)$ the CLSM and $(b)$ the AFM. Image areas: (a) $80 \times 80 \mu \mathrm{m}^{2}$, bar: $10 \mu \mathrm{m},\left(\right.$ b) $24 \times 24 \mu \mathrm{m}^{2}$, bar: $3 \mu \mathrm{m}, 25$ pixels $/ \mu \mathrm{m}$. 


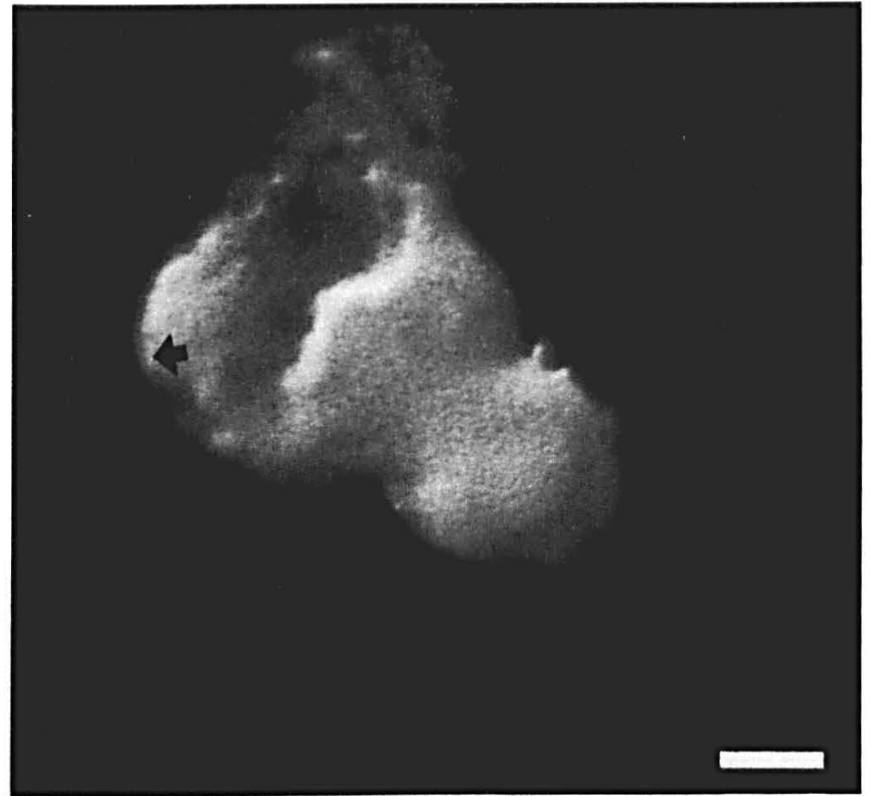

(a)

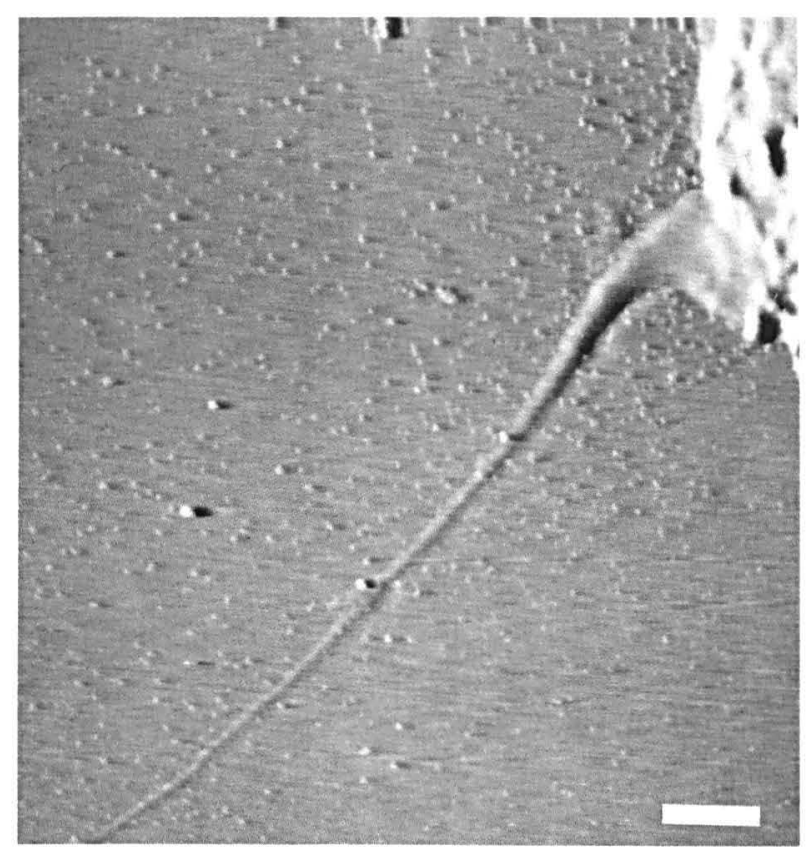

(b)

Figure 3. Images of a conjugate of NK- and K562-cell stained with BODIPY 581/591 Phalloidin recorded with (a) the CLSM and $(b)$ the AFM. The smaller cell in the lower right corner of $(a)$ is the NK-cell. The AFM image $(b)$ is taken at the position indicated by the arrow (a). Image areas: $(a) 40 \times 40 \mu \mathrm{m}^{2}$, bar: $5 \mu \mathrm{m}$, (b) $24 \times 24 \mu \mathrm{m}^{2}$, bar: $3 \mu \mathrm{m}, 25$ pixels $/ \mu \mathrm{m}$.

The difference in appearance of confocal images in figures 3 and 4 is due to the fact that in figure 4 only the lower half of the focal sections are used to generate the 'simulated fluorescence' image. This allows one to 'look inside' the cell.

The CLSM images in figures 2, 3 and 4 lack somewhat the highly structured filamentous appearance of cytoskeletal components which one normally associates with confocal images. The most important reason is that the cells were dried. During the drying process the cells collapse and the already dense network of actin filaments in K562- and NK-cells becomes even more dense, resulting in confocal images in which it is hard to discriminate actin filaments. A cell with a more pronounced cytoskeleton, even when dried, is the monkey kidney cell. In figure 5 images are shown of monkey kidney cells recorded in air and under liquid. The CLSM reveals the filamentous nature of the cytoskeleton both on air dried cells and cells imaged under PBS (figure 5(a) and $5(c)$ ). Figure $5(b)$ shows the AFM image of the bottom part of the monkey kidney cell in figure 5(a). The large structure at the top-center of figure $5(b)$ is part of the nucleus, as was confirmed by the CLSM data. The particles on and near the cell are probably small salt crystals. Below the nucleus the cytoskeleton is clearly revealed, its height ranging from 50 to $150 \mathrm{~nm}$. A high degree of correlation is apparent between images in figure $5(a)$ and $5(b)$. In addition, smaller filaments are visible in the AFM image which are not visible in the confocal image. The AFM image of figure 5(d) (of the right cell in figure $5(c)$ ) is recorded under liquid. Also in this case the actin filaments are well resolved. Again, a one-to-one correlation is evident, apart from the filament reaching towards the other cell in figure 5(c). During scanning the AFM tip has removed this filament from the glass substrate (see arrow in figure $5(d)$ ). Also, the white bumps on the cell have a streakiness in the direction of scanning, indicating that material is being removed by the tip. This illustrates one of the major problems and challenges of imaging biological samples under liquid with the AFM; imaging with very low imaging forces in order not to destroy the sample. Measurements and calculations on the deformation of various materials indicate that imaging forces of about $10^{-11} \mathrm{~N}$ should be used to image non-destructively biological samples with nanometer resolution [23]. This implies an imaging force two orders of magnitude lower than routinely used while imaging under liquid. Cantilevers with a lower spring constant, increased stability of the AFM and slower scanning could be solutions to this problem.

A closer look at the AFM images shows that a 'blanket of noise' is superimposed on the images. This is due to the fact that the vibration isolation of the confocal microscope, sufficient for CLSM operation, is not adequate for optimal operation of the AFM. The vibrations which contribute to the noise in the AFM image originates from different sources. Firstly, the 
(a)

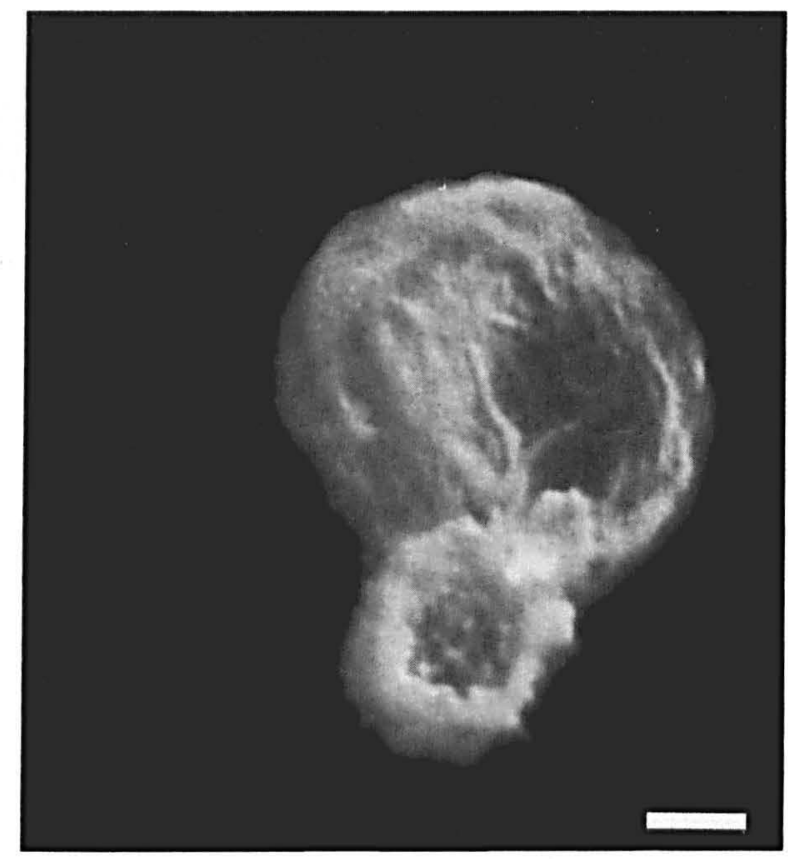

(b)

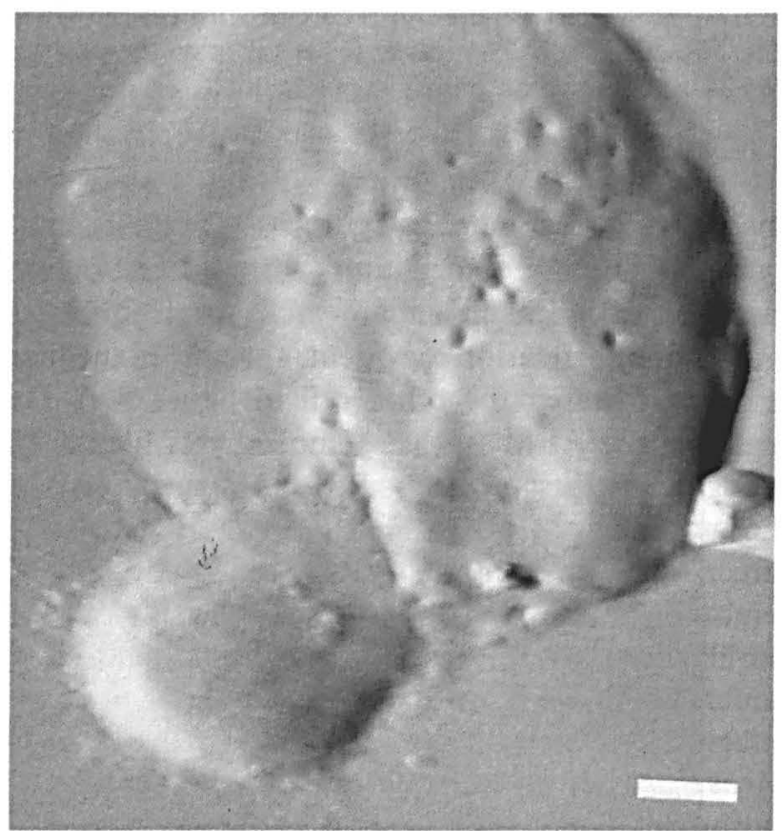

(c)

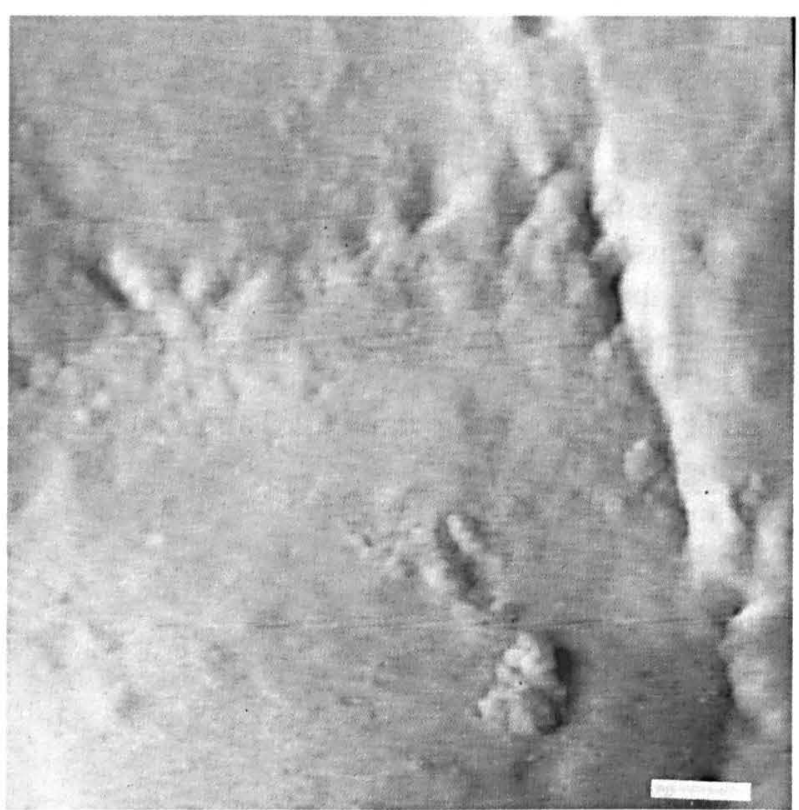

Figure 4. Images of a conjugate of NK- and K562-cell stained with BODIPY 581/591 Phalloidin recorded with (a) the CLSM and $(b, c)$ the AFM. The small cell at the bottom is the NK-cell. Image areas: $(a) 40 \times 40 \mu \mathrm{m}^{2}, 5 \mu \mathrm{m},(b) 24 \times 24 \mu \mathrm{m}^{2}$, bar: $3 \mu \mathrm{m}$, 25 pixels $/ \mu \mathrm{m},(c) 8 \times 8 \mu \mathrm{m}^{2}$, bar: $1 \mu \mathrm{m}, 75$ pixels $/ \mu \mathrm{m}$.

scanning sample stage vibrates with an amplitude of about $100 \mathrm{~nm}$ in the $70-100 \mathrm{~Hz}$ range. As mentioned in the material and methods section, this can be reduced by a factor of 10 by clamping down the stage to the base plates of the optical microscope and the AFM (see figure $1(c)$ ). Another source is the fan of the air-cooled krypton-argon laser. A better isolation or a water-cooled laser system would solve this problem. Placed on a vibration isolation table, the resolution of the stand-alone
AFM in the $z$-direction is better than $0.1 \mathrm{~nm}$ (the detection limit of the optical beam deflection system is $0.01 \mathrm{~nm}$ in a bandwidth of $10 \mathrm{kHz}$ ), as is illustrated by images of mica showing the hexagonal molecular packing [24]. In addition to the vibrations and the deformation of the surface as pointed out above, a feature inherent in the operation of the AFM is the convolution between surface topography and the tip. Due to the finite size of the tip (typically 30 to $50 \mathrm{~nm}$ ), objects lying on a flat 


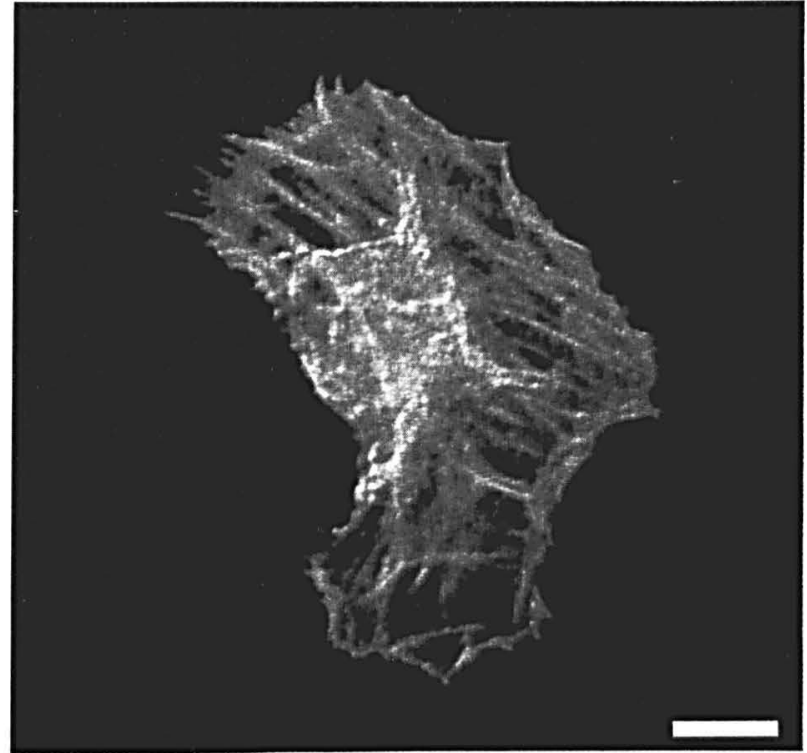

(a)

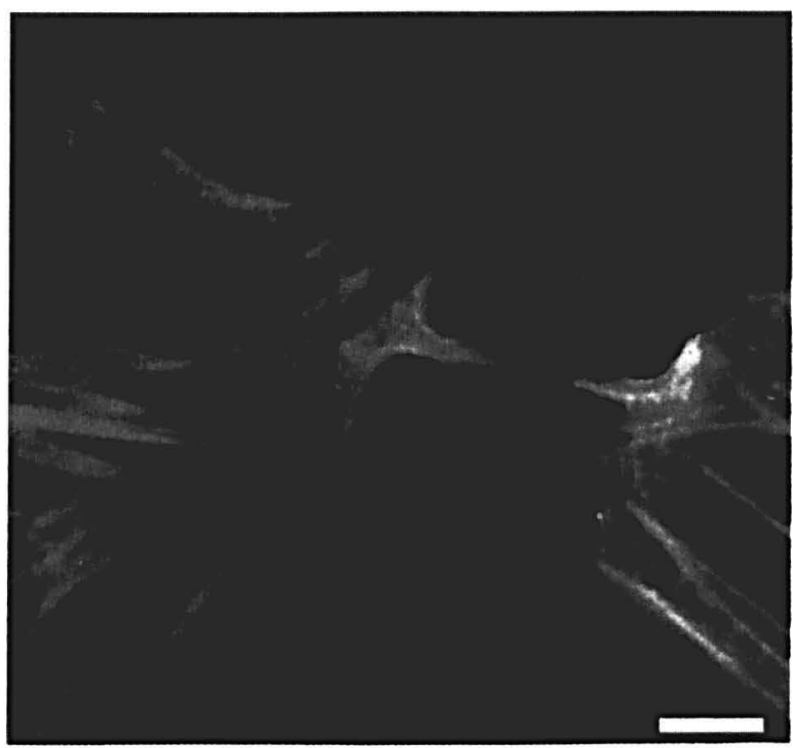

(c)

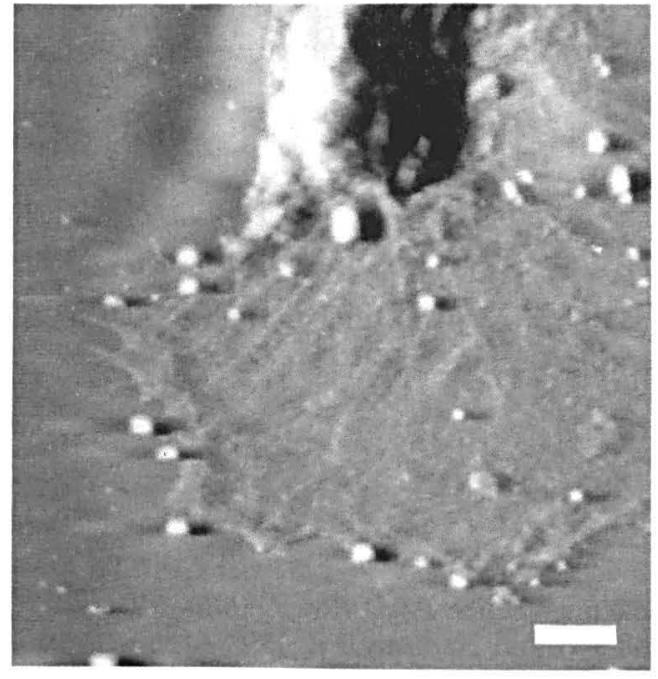

(b)

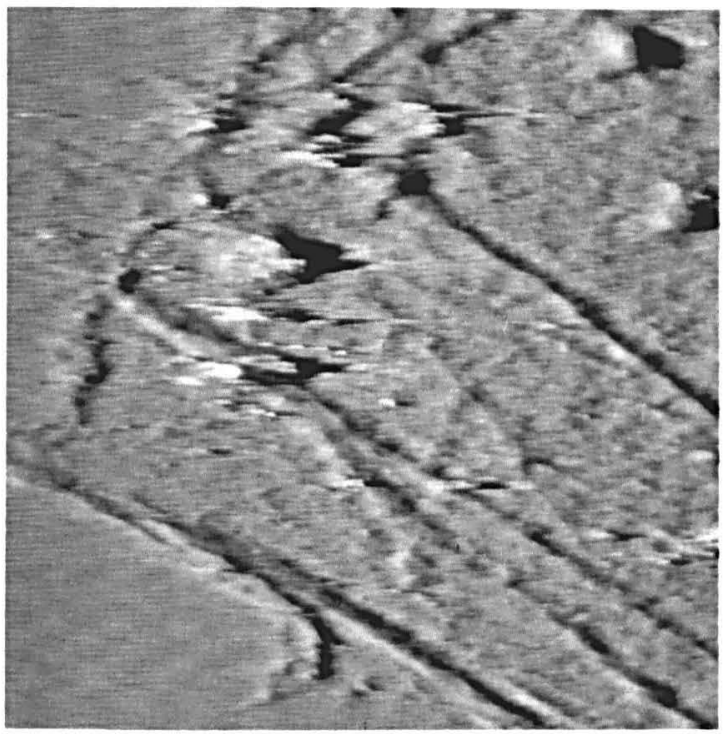

(d)

Figure 5. Images of monkey kidney cells stained with BODIPY 581/591 Phalloidin recorded with $(a, c)$ the CLSM and $(b)$ the AFM. Images $(a, b)$ were obtained in air and $(c, d)$ were obtained under liquid. Image area: $(a) 80 \times 80 \mu \mathrm{m}^{2}$, bar: $10 \mu \mathrm{m}$, (b) $22 \times 22 \mu \mathrm{m}^{2}$, bar: $3 \mu \mathrm{m}, 14$ pixels $/ \mu \mathrm{m},(c) 40 \times 40 \mu \mathrm{m}^{2}$, bar: $5 \mu \mathrm{m},(d) 15 \times 15 \mu \mathrm{m}^{2}$, bar: $2 \mu \mathrm{m}, 33$ pixels $/ \mu \mathrm{m}$. (b) has been filtered to remove a periodic vibration. Arrow in $(d)$ : see text.

surface will appear larger in the AFM image. Because of this, the resolution in an AFM depends both on the tip size and the surface morphology.

In this study we have used fixed cells which were imaged in air and in liquid. In future experiments we will further exploit the possibility to obtain AFM as well as CLSM images in a liquid environment. This should allow us to study the dynamics of the cytotoxic interactions between cells under physiological conditions.

\section{Conclusions}

A system combining a stand-alone AFM and a confocal laser scanning microscope has been presented. The combination enables one to study the cell surface structure with the high-resolution AFM and the internal structure and the membrane with the confocal microscope. Results on K562-cells, conjugates of NK- and K562-cells and monkey kidney cells show a high 
correlation between images obtained with the AFM and with the CLSM. Also differences have been observed. This enables one to obtain a more complete picture of an object.

\section{Acknowledgments}

This research was supported by the Dutch Organization for Scientific Research NWO.

\section{References}

[1] Binnig G, Quate C F and Gerber Ch 1986 Atomic force microscope Phys. Rev. Lett. 56 930-33

[2] Hoh J H and Hansma P K 1992 Atomic force microscopy for high-resolution imaging in cell biology Trend. Cell Biol. 2 208-13

[3] Keller D, Chang L, Luo K, Singh S and Yorgancioglu M 1992 Scanning force microscopy of cells and membrane proteins Proc. SPIE 1639 91-101

[4] Henderson E, Haydon P G and Sakaguchi D S 1992 Actin filament dynamics in living glial cells imaged by atomic force microscopy Science 257 1944-6

[5] Wilson T (ed) 1990 Confocal Microscopy (London: Academic)

[6] Hipp M, Bielefeldt H, Colchero J, Marti O and Mlynek J 1992 A stand-alone scanning force and friction microscope Ultramicroscopy 42-44, 1498-503

[7] Putman C A J, Hansma H G, Gaub H E and Hansma P K 1992 Polymerized LB films imaged with a combined atomic force microscope-fluorescence microscope Langmuir 8 3014-19

[8] Sarid D, Pax P, Yi L, Howlls S, Gallagher M, Chen T, Elings V and Bocek D 1992 Improved atomic force microscope using a laser diode interferometer Rev. Sci. Instrum. 63 3905-8

[9] Meyer G and Amer N M 1988 Novel optical approach to atomic force microscopy Appl. Phys. Lett. 53 2400-2

[10] Radosevic K, Garritsen H S P, Van Graft M, De Grooth B G and Greve J 1990 A simple and sensitive flow cytometric assay for the determination of the cytotoxic activity of human natural killer cells J. Immunol. Methods 135 81-9

[11] Bolhuis R L H, Van Griend R J and Ronteltap C P M 1984 Clonal expansion of human B73.1-positive natural killer cells or large granular lymphocytes exerting strong antibody-dependent and -independent cytotoxicity and occasionally lecti-dependent cytotoxicity Nat. Immun. Cell Growth Regul. 3 61-72

[12] Albrecht T R, Akamine S, Carver T E and Quate C F 1990 Microfabrication of cantilever styli for the atomic force microscope J. Vac. Sci. Technol. A 8 3386-96

[13] Purchased from Park Scientific Instruments, Sunnyvale, CA, USA

[14] Putman C A J, De Grooth B G, Van Hulst N F and Greve J 1992 A detailed analysis of the optical beam deflection technique for use in atomic force microscopy J. Appl. Phys. 72 6-12

[15] Weisenhorn A L, Hansma P K, Albrecht T R and Quate C F 1987 Forces in atomic force microscopy in air and water Appl. Phys. Lett. 54 2651-3

[16] Putman C A J, Van der Werf K O, De Grooth B G, Van Hulst N F, Greve J and Hansma P K 1992 A new imaging mode in atomic force microscopy based on the error signal Proc. SPIE 1936 198-204

[17] Knebel W, Quader H, Wijnaendts-van-Resandt R and Engelhardt H 1989 Application of confocal laser scanning microscopy in plant biology Ber. Bunsenges. Phys. Chem. 93 380-6

[18] Carlini A and Wilson T 1989 The role of pinhole size and position in confocal imaging systems Scanning Imaging ed T Wilson (Proc. SPIE 809 97-100)

[19] Van der Voort H T M and Brakenhoff G J 1988 Modelling of 3-D confocal imaging at high numerical aperture in fluorescence Scanning Imaging ed T Wilson (Proc. SPIE 1028 39-44)

[20] Brakenhoff G J, Wijnaendts R W, Engelhardt J, Knebel W and Van der Voort H T M 1988 Inverted confocal microscopy for biological and material applications Scanning Imaging ed T Wilson (Proc. SPIE 1028 169-72)

[21] Van der Voort H T M, Brakenhoff G J and Baarslag M W 1989 3-Dimensional visualization methods for confocal microscopy J. Microsc. 153 123-32

[22] Putman C A J, Van der Werf K O, De Grooth B G, Van Hulst N F, Segerink F B and Greve J 1992 Atomic force microscope with integrated optical microscope for biological applications Rev. Sci. Instrum. 63 1914-17

[23] Weisenhorn A L, Khorsandi M, Kasas S, Gotzos V and Butt H-J 1993 Deformation and height anomaly of soft surfaces studied with an AFM Nanotechnology $\mathbf{4}$ in press

[24] Putman C A J, Van der Werf K O, De Grooth B G, Van Hulst N F and Greve J 1993 Stand-alone atomic force microscope featuring large scan, friction measurement, atomic resolution and capability of liquid operation Proc. SPIE 1855 in press 